\title{
Mathematical Morphology And Wavelet Transformation Based Moving Object Detection Algorithm
}

\author{
Fei Yu \\ Xi'an University of Technology \\ Xi'an, China \\ sxyufei1980@163.com
}

\author{
Jianqiang Feng \\ Xi'an University of Technology \\ Xi'an, China \\ sxfjq1980@163.com
}

\begin{abstract}
Moving object detection (MOD) is gaining extensive attention. On the basis of introduction of three conventional ways for MOD, in order to overcome the disadvantage of inter-frame difference method can not completely extract all the relevant points for the moving object, a new moving object detection (MOD) algorithm based on mathematical morphology (MM) and wavelet transformation (WT) is proposed. Firstly, on the basis of description of the basic theory of WT and MM, the detail part of the detected image is achieved by utilization of WT. Then, the inverse wavelet transformation is applied to reconstruct the signal. It finally gets the detection target by means of open and close calculation from MM. In order to evaluate the performance of the proposed algorithm in the paper, clear images fitting for computer digital image processing are selected to be detected. The experimental results show that the presented algorithm can detect the whole moving target, and satisfy the real-time requirement.
\end{abstract}

Keywords-moving object detection; mathematical morphology; wavelet transformation; inter-frame difference method; frame detection

\section{INTRODUCTION}

With the increase of the people's demands for video recognition, moving object detection (MOD) has been gained an extensive attention as an extremely important research point for sport detection, video surveillance, etc. For the aim of MOD, it can improve the automation level of visual system by means of a variety of sensors. The conventional ways for MOD mainly include the following: 1) Optical flow ${ }^{[1]}$. Optical flow method is a method of the utilization of motion estimation segmentation to track the moving targets. This method can achieve goals for sports detection and tracking under the condition of no prior knowledge of background area; it also can be applied to the camera movement, but its calculation is very large, and is very sensitive to external noise. 2) Inter-frame difference ${ }^{[2]}$. Inter-frame difference method is to extract image area in the continuing two or three adjacent frames based on temporal difference of pixels and thresholding. Consecutive frame difference has a good adaptability to dynamic environment, but the detection location is not accurate. In addition, the external rectangle for moving object is stretched in the direction of sport and can not completely extract all the relevant points for the moving object. And it may produce a cavity in the internal of the moving object. 3) Background subtraction ${ }^{[3]}$. This method detects moving area by means of the difference between current image and the background image. Its significant advantage is detection the location accurate, and has a rapid speed. Additionally, it needs little detection information. The deficiency of background subtraction method is that it is easily affected by the change of light conditions; and it needs an update mechanism for background image in an uncontrolled environment. In addition, this method requests a small change of the background for the whole scene and an accurate modeling for the establishment of the background. Thus it can be seen that various detection methods have their own advantages and disadvantages.

In order to overcome the shortcomings of inter-frame method and improve real-time performance of moving object detection, this paper presents a mathematical morphology and wavelet transformation based moving object detection algorithm. The structure of the paper is as follows: the paper begins with the introduction of existing situation for moving object detection; then, it analyzes the detection principle of inter-frame method in section 1; the principle of frame detection method is discussed in section 2; the basic theory of mathematical morphology and wavelet transformation utilized in the paper are discussed in section 3; section 4 gives the detail detection steps; and with respect to discussion of the experiment results is completed in section 5 . The last section is the conclusions obtained in the research.

\section{DeteCtion PRinciple OF FRAME DETECTION METHOD}

The basic principle of inter-frame method [4] is firstly to achieve the information of continuously adjacent two or three frames; then, the temporal differential and thresholding method based on pixel is utilized to extract the motion area. In general, the method includes three steps: pre-processing, background recovering, and moving object extraction. The aim of pre-processing is to eliminate the effect of noise before extraction. Thus, background recovering can apply the statistical information in terms of pixel values.

Define image sequence is $I(x, y, i)$, where $x$ and $y$ represent the coordinate in space, $i(=1,2,3, \cdots, N)$ indicates the frame, $N$ is the total number of frames. The calculation method and judgment rule can be written in the following and shown in (1) and (2) respectively.

$$
S(i, j, t)=|I(i, j, t)-I(i, j, t-1)| \text {. }
$$




$$
H(i, j, t)=\left\{\begin{array}{l}
1 \text { if }|I(i, j, t)-I(i, j, t-1)|>t h_{1} \\
0 \text { if }|I(i, j, t)-I(i, j, t-1)| \leq t h_{1}
\end{array}\right.
$$

where $S(i, j, t)$ represents the gray difference value between time $t-1$ and time $t$ for the detection frame; $H(i, j, t)$ indicates ultimate frame difference value, 1 signifies that the pixel is moving, 0 demonstrates that the pixel is static; threshold1 represents the threshold value. The merits of this method are rapid detection speed and good environmental adaptability. However, the method can not extract all the related points.

\section{DESCRIPTION OF THE BASIC THEORY}

Wavelet transformation has an advantage of real-time and robustness; thus mathematical morphology can be able to fill the empty hole, remove noise and make information completely. Hence, the fusion of the two algorithms on the basis of the method of inter-frame can avoid the defects of traditional detection methods to some extent.

\section{A. Wavelet Transformation}

The wavelet transformation (WT) is scalable and translatable in discrete steps, resulting in a discrete sampling in the time scale representation. Thus, the calculation formula of WT can be written as the following [5].

$$
\psi_{j, k}(t)=\frac{1}{\sqrt{s_{0}^{j}}} \psi\left(\frac{t-k \tau_{0} s_{0}^{j}}{s_{0}^{j}}\right) .
$$

where $j$ and $k$ represent the scale and shift respectively, and $s_{0}$ defines the dilation step. Thus we can describe the wavelet transformation as follows [5]:

$$
X(j, k)=\sum_{j} \sum_{k} x(k) 2^{-\frac{j}{2}} \psi\left(2^{-j} n-k\right) .
$$

The WT can be calculated by passing the signal through a filter bank, where the signal is simultaneously passed through both a low pass filter and a high pass filter, which act as the scaling and wavelet function, respectively. Repeating this process produces a binary tree representation of the filter bank, where each node in the tree represents different time / frequency localizations, and each level in the binary tree is a different decomposition level.

This representation is shown in Figure 1 and Figure 2. Pass the signal through a low pass filter results in the production of a series of approximate coefficients, while the detail coefficients are given from passing the signal through the high pass filter. This can be seen [6] from the following equations:

$$
Y_{\text {high }}[k]=\sum_{n} x[n] g[2 k-n]
$$

$$
Y_{\text {low }}[k]=\sum_{n} x[n] h[2 k-n]
$$

where $h[\cdot]$ and $g[\cdot]$ are weighting factors that form the low pass and high pass filters, respectively. In order to keep the output data rate equal to the input data rate $\mathrm{T}$ instead of it being doubled after passing through each filter. Apply a factor of two to the high and low pass filters, denoted by $2 k$ in (5) and (6), effectively subsampling the output of each filter by 2 . This process described one iteration, or level, of the DWT calculation algorithm. The signal can be further decomposed by repeating the process using the approximate coeffcients as input to the next level of high and low pass filters. The maximum number of wavelet decomposition levels that can be applied to a signal is related to the length of the signal, $M=L^{2}$, where $M$ is the length of the signal and $L$ is the maximum decomposition level.

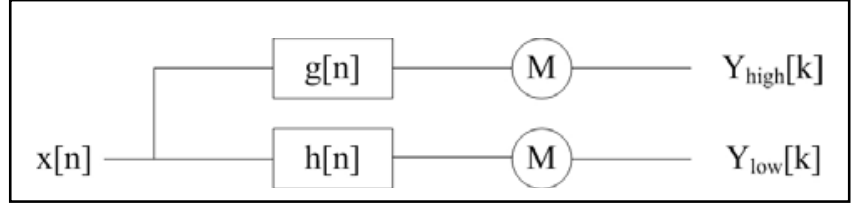

Figure 1. Description of WT.

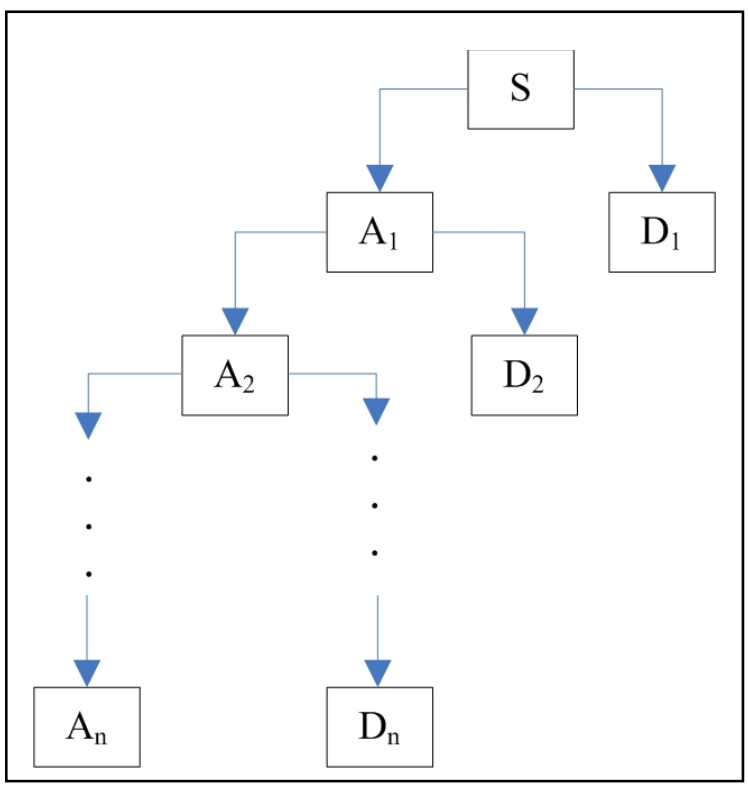

Figure 2. Binary tree representation of WT filter bank.

The extracted coefficients represent the decomposed signal at different resolutions: detailed information is produced by the high pass filter at a low scale and a coarse representation produced by the low pass filter at a high scale. These coefficients, produced by the WT, give a timefrequency representation of the signal in a compressed fashion, representing how the energy of the signal is distributed in the time-frequency domain.

\section{B. Mathematical Morphology}

Morphological filters and operations are non-linear signal transformation tools that modify shapes of signals $[7,8]$. The 
technique is based on mathematical morphology (MM), originally developed by Matheron [9] and Serra [10]. MM is developed from set theory and integral geometry, and derives its name because it deals with the shape of signals. Unlike Fourier transform or wavelet transform, which extract frequency information from signals, MM works exclusively in time domain and is useful in de-noising signals and images.

The basis of morphological filter or morphological operation is a function known as structuring element (SE) that is used to process a given signal. Morphological transform is able to split a complex signal into several subsignals with different physical significance. The applications of MM, including filtering, involve judicious use of such sub-signals to achieve the final objective.

Dilation and erosion are two basic operations in MM. Based on these two; several other compound operators such as closing, opening, hit or miss, top-hat transform etc are defined [11]. Signals in image are usually multi-dimensional. The most commonly used operations for such signals are dilation, erosion, closing and opening. The definitions of these are presented below.

Let $a(n)$ and $b(m)$ be an input signal and an SE, defined in the domains, $D_{a}\left(a_{0}, a_{1}, \ldots, a_{n}\right)$ and $D_{b}\left(b_{0}, b_{1}, \ldots, b_{m}\right)$ respectively, with $n>m$, where $n$ and $m$ are integers. Then the dilation of $a(n)$ by $b(m)$, denoted by $(a \oplus b)$ is defined as:

$$
y_{d i l}(n)=(a \oplus b)(n)=\max \{a(n-m)+b(m)\},
$$

where $0 \leq(n-m) \leq n, m \geq 0$.

Similarly, erosion of $a(n)$ by $b(m)$, denoted by $(a \otimes b)$ is defined as:

$$
y_{\text {ero }}(n)=(a \otimes b)(n)=\min \{a(n+m)-b(m)\},
$$

where $0 \leq(n+m) \leq n, m \geq 0$.

Using the two basic operations described in (7) and (8), two derivative operations called closing and opening are defined. The closing of $a(n)$ by $b(m)$, denoted by $(a \bullet b)$, is defined as erosion of the dilated signal $(a \otimes b)$ by $b$ :

$$
y_{\text {close }}(n)=(a \bullet b)(n)=((a \oplus b) \otimes b)(n) .
$$

Similarly, opening of $a(n)$ by $b(m)$, denoted by ( $a \circ b)$, is defined as dilation of the eroded signal $(a \otimes b)$ by $b$ :

$$
y_{\text {open }}(n)=(a \circ b)(n)=((a \otimes b) \oplus b)(n)
$$

Opening generally smoothes the sharp edges in a contour, whereas closing fills narrow valleys and gaps in a contour [12].

\section{The Proposed Method IN The PAPER}

In terms of the principle of inter-frame method, integration the vantages of WT and MM, an improved algorithm for moving object detection is proposed in the paper, the detection steps of which is shown in Figure 3.

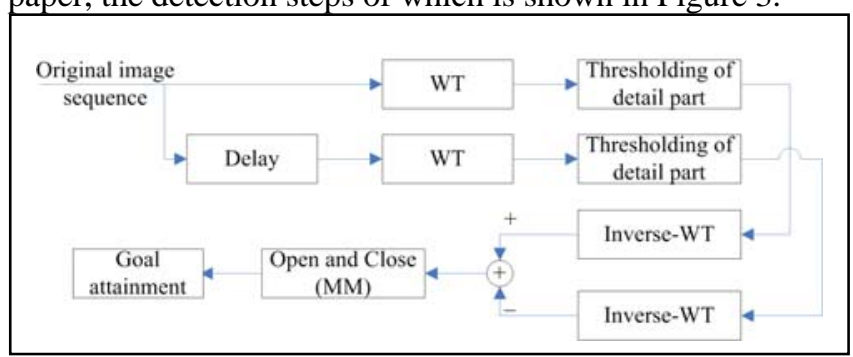

Figure 3. Description of the proposed algorithm in the paper.

From Figure 3 we can see that the proposed algorithm in the paper takes full advantage of WT and MM. For WT, we utilize the detail part of original image information; and for MM, we apply the calculation of open and close.

\section{EXAMPLE AND DISCUSSIONS}

In order to evaluate the performance of the proposed algorithm in the paper, clear images fitting for computer digital image processing are gotten firstly and shown in Figure 4 to Figure 6.

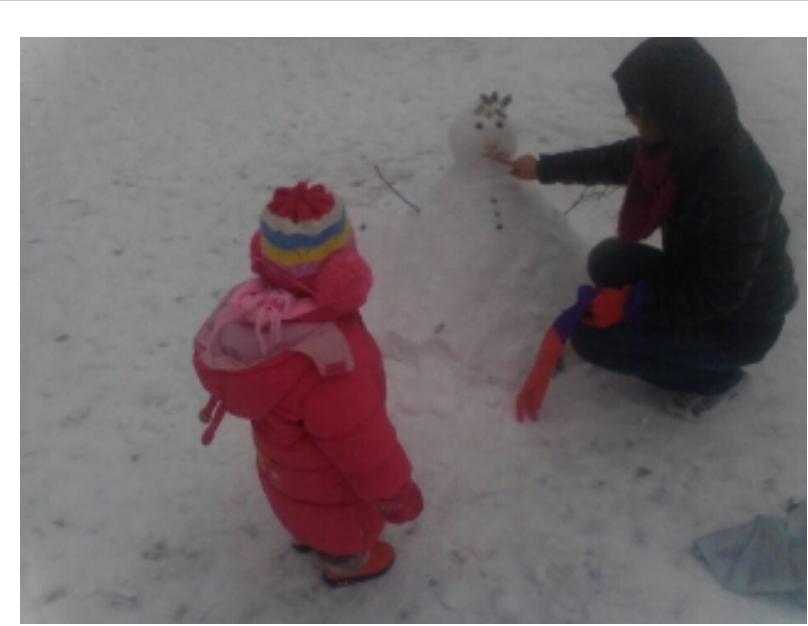

(a) The first frame

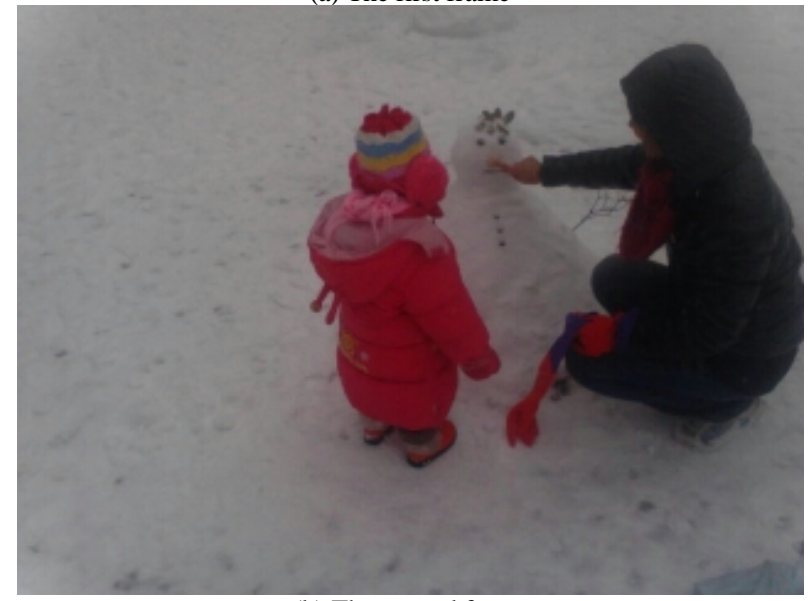

(b) The second frame

Figure 4. Selection of two frames in the original images. 


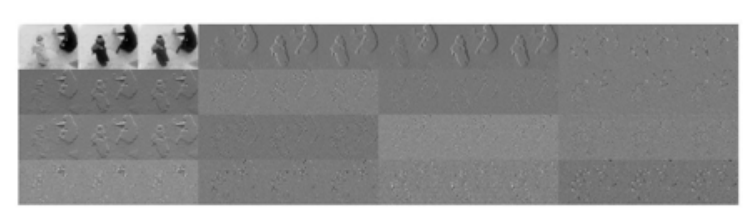

(a) The first frame after WT

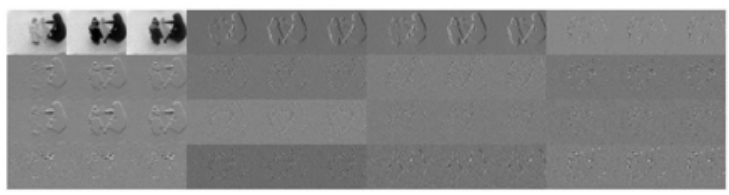

(B) The second frame after WT

Figure 5. The two frames in the original images after WT.

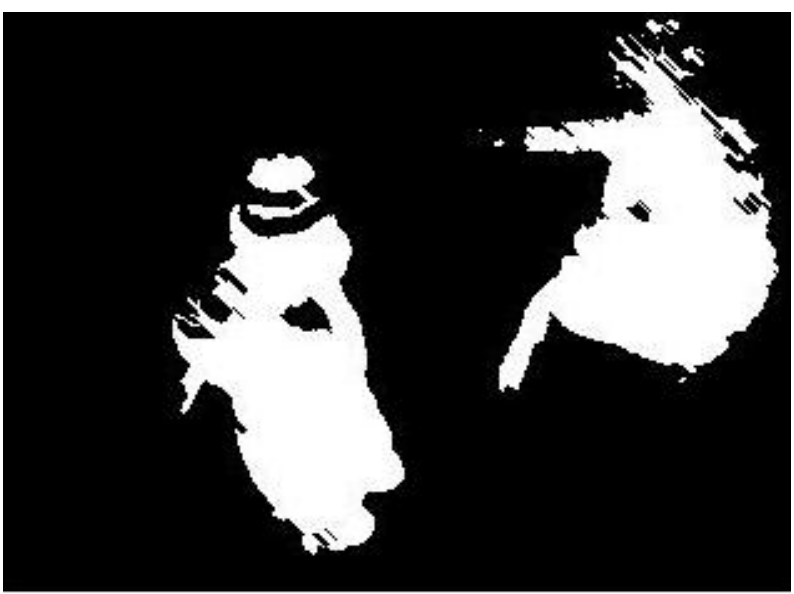

(a) The first frame

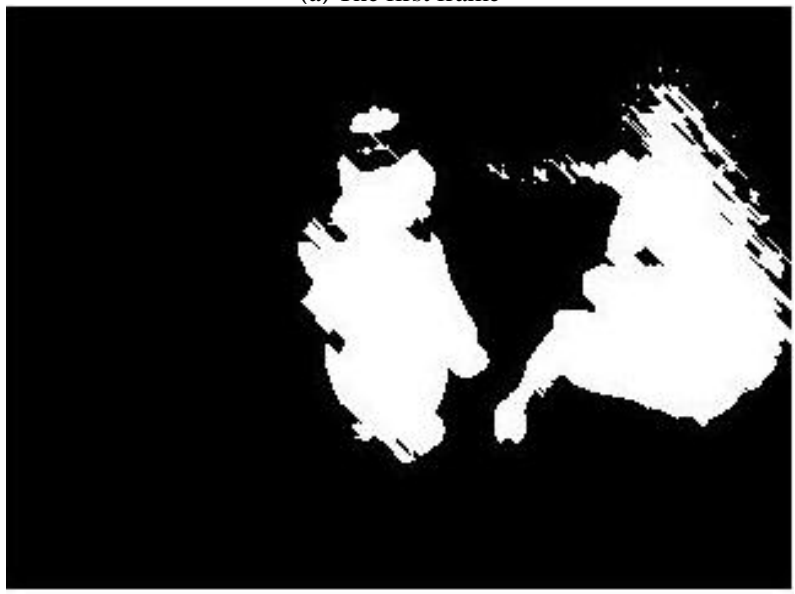

(b) The second frame
Figure 4 describes the two frames in collected pictures. Figure 5 shows the results of the two frames in the original images after WT. Figure 6 gives the final detection results of the two frames after MM calculation of close and open. The detection results show that the proposed algorithm in the paper utilizes the multi-scale characteristics of wavelet. In addition, it greatly reduces the influence of the noise to moving object detection by means of MM.

\section{CONCLUSIONS}

For the defect of conventional detection method, a moving object detection algorithm based on MM and WT integrating inter-frame method is proposed. The experiment test shows that the proposed algorithm is fit for tracking complex moving object, and has a good and real-time detection performance.

\section{REFERENCES}

[1] W. Wang, Z .X. Su, J. S. Pan, Y. Wang, and R. M. Sun, "Robust optical flow estimation based on brightness correction fields”, J. Zhejiang Unversity (Sci. C), vol. 12, No. 12, pp. 1010-1020, 2011.

[2] B. Thomas, B. Andrés, P. Nils, and W. Joachim, "High accuracy optical flow estimation based on a theory for warping”, Lecture Notes in Computer Science, Vol. 3024, pp. 25-36, 2004.

[3] Massimo Picardi, "Background subtraction techniques: a review", 2004 IEEE Conf. Syst. Man and Cyber., Vol. 7, The hague, pp. 30993104, 2004.

[4] S. F. Wu and J. Kittler, "A differential method for simultaneous estimation of rotation, change of scale and translation”, Signal processing: Image communication, Vol. 2, no. 1, pp. 69-80, 1990.

[5] Stéphane Mallat, A Wavelet Tour of Signal Processing: The Sparse way. Academic Press, 2009.

[6] M. Shensa, "The discrete wavelet transform: wedding the a trous and Mallat algorithms”, IEEE Trans. Sign. Proc., Vol. 40, no. 10, pp. 2464-2492, 1992.

[7] P. Maragos and R. Schafer, "Morphological filters-part I: Their settheoretic analysis and relations to linear shift-invariant filters," IEEE Trans. Acoust., Speech, Signal Process., vol. 35, no. 8, pp. 1153-1169, 1987.

[8] P. Maragos and R. Schafer, "Morphological filters-part II: Their relations to median, order-statistic, and stack filters," IEEE Trans. Acoust., Speech, Signal Process., vol. 35, no. 8, pp. 1170-1184, Aug. 1987.

[9] G. Matheron, Random Sets and Integral Geometry. New York: John Wiley, 1975.

[10] J. Serra, Image Analysis and Mathematical Morphology. New York: Academic Press, 1982.

[11] G. Li, M. Zhou, Y. Luo, and Y. Ni, "Power quality disturbance detection based on mathematical morphology and fractal technique," in Transmission and Distribution Conference and Exhibition: Asia and Pacific, 2005IEEE/PES, 2005, pp. 1-6.

[12] R. C. Gonzalez and R. E. Woods, Digital Image Processing, 2nd ed. New Jersey: Prentice Hall, 2002.

Figure 6. Detection results after close and open. 